\title{
MORPHOLOGICAL AND MOLECULAR CHARACTERIZATION OF A NEW SPECIES OF ATLANTIC STALKED BARNACLE (SCALPELLIFORMES: POLLICIPEDIDAE) FROM THE CAPE VERDE ISLANDS
}

\author{
Quinteiro, J. ${ }^{\text {a }}$, Rodríguez-Castro, J. ${ }^{\text {, }}$ López, P. ${ }^{\text {, }}$ López-Jurado, L.F. , González- \\ Henrquez, N. ${ }^{d}$, Rey-Méndez, M. ${ }^{a}$
}

\footnotetext{
a Laboratorio de Sistemática Molecular (Unidad Asociada CSIC-IIM), Departamento Bioquímica e Bioloxía Molecular. CIBUS, Universidade de Santiago de Compostela, 15782 Santiago de Compostela (A Coruña), Spain.

b Proyecto Naturalia. Boa Vista, Cape Verde Islands, Cape Verde.

c Facultad de Ciencias Del Mar, Universidad de Las Palmas de Gran Canaria, Campus Universitario de Tafira, 35017 Las Palmas de Gran Canaria, Spain.

d Instituto Canario de Ciencias Marinas, Gobierno de Canarias, Apartado 56, 35200 Telde Gran Canaria, Spain.
}

\begin{abstract}
The taxonomy of pedunculate cirripedes belonging to the genus Pollicipes has essentially remained unchanged since Charles Darwin described them in his exhaustive work on the Cirripedia. This genus includes three species of stalked barnacles: Pollicipes pollicipes in the north-eastern Atlantic, P. polymerus in the north-eastern Pacific and P. elegans in the centraleastern Pacific. However, a population genetics analysis of $P$. pollicipes suggested the presence of a putative cryptic species collected from the Cape Verde Islands in the central-eastern Atlantic. This study examines the morphology of these genetically divergent specimens and compares them with that of representative Atlantic samples of the biogeographically closely related $P$. pollicipes and with the poorly described $P$. elegans. Molecular data, including mitochondrial COX1 and nuclear ribosomal interspaces sequences, were obtained for all species of the genus Pollicipes. Morphological distinctiveness, diagnostic characters, congruent divergence level and monophyletic clustering, at both nuclear and mitochondrial loci support the taxonomic status of this new species, Pollicipes darwini.
\end{abstract}

